\title{
Timing of referral for consideration of surgery to the upper limb in patients with spasticity after stroke
}

\section{Conceptual paper}

Stroke may result in significant disability to the affected individual. Each year there are 152,000 strokes in the UK resulting in 1.2million stroke survivors? Stroke survivors may have a disability of the lower limb and/or the upper limb leaving one third of subjects dependent on others (Stroke association) resulting in a significant burden to families and social care systems.

The upper motor neuron lesion may cause spasticity in the affected limbs. The pathophysiological processes causing spasticity are not fully understood, however there is muscle imbalance, impaired movement, abnormal joint posture and functional impairment. The altered function of the upper limb may result in limitations in the activities of daily living.

At the acute phase (minutes to hours) after a stroke the treatment is focused on minimizing the extent of the neurological damage by restoring and enhancing blood supply to the affected area of the central nervous system. The sub-acute phase of stroke (days to weeks) treatment is focused on helping the patient to recover from the cerebrovascular event and prevention of further episodes. During this phase the limb priority is the prevention of deformity due to joint contractures from spasticity and recruitment of the affected limb for functional tasks using techniques including constraint-induced movement therapy. Static preventative splinting techniques and chemo-denervation are the main treatment interventions introduced at this stage to assist in upper limb rehabilitation in patients with hypertonia. Treatment is individualized and depends on the degree of physical and cognitive impairment the functional potential and the general medical condition of the patient.

Neurologic recovery is not expected to continue for more than 5months following the cerebrovascular event (Jørgensen HS et al, 1995). Functional recovery may continue to improve with therapy interventions but there is no significant change in the patient's neurological status.

In the non-functional limb with no volitional control hygiene and difficulties in dressing are common problems facing patients and carers. Surgical intervention may help in the non-functional spastic limb either in the prevention of contractures or in the correction of contractures. Procedures aimed at reducing deforming forces across joints include muscle-tendon lengthening, tendon transfers, and neurectomies.

In the non-functional shoulder the spastic pectoralis major, latissimus dorsi, teres major and subscapularis muscles can be released ${ }^{1}$ reducing pain, improving passive range of motion, axillary hygiene and ease of washing and dressing. Upper limb tone reduction may improve lower limb function with improved gait cadence and reduced energy expenditure of walking. Selective motor neurectomies in the infra-clavicular brachial plexus may achieve the same result in patients with no contractures or can be supplemented with splinting in mild contractures. In the non-functional elbow, neurectomy of the
Volume 9 Issue 5 - 2019

\author{
Petros Mikalef, Rizwana Imran, Dominic \\ Power, Peter Harding \\ University Hospital Birmingham NHS Foundation Trust, UK
}

Correspondence: Rizwana Imran, University Hospital Birmingham NHS Foundation Trust, 58 Hollie Lucas Road Kings Heath Birmingham United Kingdom B 3 0QN, UK, Tel 7946554206, Email rizwana.imran@nhs.net

Received: July 14, 2019 | Published: September 02, 2019

musculocutaneous nerve can improve elbow position, reduce pain, improve hygiene and ease of dressing. Satisfactory outcomes have been described. ${ }^{2,3}$

In the nonfunctional wrist transfer of the flexor digitorum superficialis to the flexor digitorum profundus (STP tendon transfer) combined with lengthening of the wrist flexors and the flexor policies longus ${ }^{4,5}$ or neurectomy of the ulnar nerve $e^{6,7}$ have provided satisfactory results.

Longstanding, severe or poorly managed spasticity may result in the development of joint contractures. ${ }^{8}$ Fixed joint deformities may be treated with serial casting but are generally resistant to therapy interventions. Washing, dressing and hygiene difficulties may be addressed surgically. Severe contractures will always require muscletendon releases to improve passive range of motion.

When the affected joint is the shoulder and there is adduction contracture with painful inferior glenohumeral subluxation, tenotomy of the pectoralis major, lattisimus dorsi and teres major can be supplemented by a biceps suspension procedure. Pain is reduced and passive range of motion is improved. ${ }^{9}$ Elbow contractures may require release of the brachioradialis, biceps and brachialis. A lateral incision avoids the elbow flexor crease where the skin quality may be poor and scar contractors may result in recurrent deformity. The results of elbow releases are reported by Keenan ${ }^{4}$ demonstrating good correction of the deformity with high patient and care satisfaction rates. Neurectomy of the musculocutaneous nerve has also been described in patients with non-functional upper limbs with satisfactory results in 29/30 (97\%) of the patients. ${ }^{2,3}$ When the forearm is involved and spastic pronation needs to be improved, release of the pronator teres is performed from its insertion onto the radius shaft.

If the wrist is involved and the deformity is not passively correctable, tendon releases are unlikely to fully correct the postural deformity. A corrective wrist fusion is indicated in such cases ${ }^{10}$ and may be combined with a proximal row carpectomy or capitate recession into the distal radius with arthrodesis as described by Louis et al. ${ }^{11}$ 
When the small finger joints are stiff causing a clenched fist deformity and a soft tissue procedure (STP transfer) fails to correct the posture, metacarpal head excision through a dorsal transverse incision is a useful strategy to improve finger position and to improve palmar hygiene. $^{12}$

In a functional limb with some volitional control rehabilitation strategies are aimed at improving the functional status of the limb. Operative interventions are sometimes indicated and are aimed at enhancing conservative interventions through rebalancing forces across joints. Chemo-denervation in targeted muscles can usually achieve this rebalancing without surgery. Repeated use of chemodenervation is frequently beneficial however patients may experience weakness, incomplete spasticity correction and have periods of high tone between treatments after the chemo-denervating agent has worn off. The resulting variable functional outcomes are frustrating for the functional patient. In such cases a surgical denervation may be considered. Joint deformity and high tone may also be managed with tendon lengthening, albeit at a cost of reduced strength.

If the shoulder is functional and spasticity needs to be reduced fractional lengthening of pectoralis major, latissimus dorsi and teres major have been described. ${ }^{13}$ Patients were reported to have reduced pain and significant improvement in active and passive shoulder motion with $92 \%$ of patients satisfied with the results of surgery.

In the functional elbow, z-lengthening of the biceps, fractional lengthening of the brachialis tendon and release of the origin of the brachioradialis ${ }^{4}$ has been the most common intervention. Satisfactory results were reported by Landi et al. ${ }^{14}$ Musculocutaneous neurectomy may provide patients with better control of elbow function, satisfactory pain relief and reduction of spasticity as demonstrated by the modified Ashworth scale and patient reported satisfaction. ${ }^{15}$

If spasticity of the pronator teres is causing pronation deformity of the forearm, fractional lengthening of the pronator teres has proved beneficial in functional limbs.

When spasticity involves the wrist and finger flexors tendon lengthening (either fractional lengthening or Z-lengthening) of the involved wrist and finger flexor muscles can improve the function of the involved hand. Active extension of the digits has to be assessed individually and may be augmented if needed using tendon transfer. ${ }^{4}$ Spasticity of the intrinsic muscles may require intervention and treatment should be tailored to the individual.

Although there are surgical options for the functional and nonfunctional upper limb after stroke, the optimum timing for surgical intervention has not been established. Traditionally operative treatment was considered when non-operative measures failed to prevent contracture formation and the aim was to improve joint position and hygiene. Unfortunately, many patients have early limited function for simple activities in the early post stroke period but because of hypertonia they lose critical parts of their range of movement and develop contractors and the limb may become nonfunctional. Judicious early surgical intervention in such cases may preserve or improve the functional status of the limb.

In the severely impaired upper limb, prolonged physiotherapy does not provide the patient with long-term functional gain. ${ }^{16}$ Prolonged hypertonia and muscle imbalance cause contractures that can be detrimental for the patient. ${ }^{8}$ In these patients earlier surgical intervention can facilitate nursing care, reduce pain and improve hygiene.
In the post-stroke functional upper limb earlier consideration of surgical intervention may provide a more permanent and consistent reduction in spasticity, maintain or enhance the functional status of the limb, reduce care dependence and the disruption of frequent hospital visits for chemo-denervation interventions.

The timing of referral for surgical assessment and the role of early surgical intervention was debated by multi-professional delegated at the recent UK Upper Limb Spasticity Meeting at the Birmingham Hand Centre in October 2017 "Pathways of care for the post-stroke patient with upper limb spasticity". The conclusion of the meeting was that earlier involvement of the surgical team is essential to optimize functional recovery and to prevent deterioration due to joint imbalances and the development of contractures.

Predictive factors for severe upper limb impairment were presented. ${ }^{17}$ Careful and standardized assessment of patients in the early post stroke phase highlights those that are at risk of contractures and should prompt surgical referral. The results of a trial exploring the role of early administration of botulinum toxin was presented demonstrating reduced elbow joint contracture development ${ }^{18}$ and the results of non-operative treatment modalities were reviewed. The optimum timing of referral for surgical management was debated with presentations from both physiotherapists and surgeons. Consensus was achieved and the conclusion of the meeting was that standardizing assessment parameters, early interventions and earlier multi-professional review would enhance the outcome for the poststoke patient.

All delegates were invited to contribute to a post-meeting survey regarding timing of referral for surgical intervention in the poststroke patient. The respondents included hand, orthopedic and plastic surgeons, neurorehabilitation consultants, physiotherapists, hand therapists and stroke physicians. There were 38 survey respondents. Delegates were able to give more than one answer to the question on timing of referral for surgical assessment: 24/38 thought optimum surgical assessment timing was 6months post-stroke; 4/38 at 12 months; $8 / 38$ after failed non-operative management; $8 / 38$ after the development of joint contractures and $5 / 38$ felt that the request for surgical assessment should be initiated by the patient.

The majority of delegates $(63.1 \%)$ agreeing that the optimum time for operative intervention should be at 6 months post-stroke matches the point at which further spontaneous neurological recovery is unlikely. This view is different to what the authors report from their own practices where typically referral for a surgical opinion is more than 3 years post-stroke with established joint contractors and attendant complications.

The adoption of the multi-professional consensus surgical assessment trigger at 6months post-stroke could reduce the onset of joint contractures in the non-functional limb. In the functional upper limb an early assessment can provide the patient with strategies to optimize function and to prevent the loss of function that frequently accompanies contracture development.

These assessments should be in a multidisciplinary setting because the management of hypertonia with chemo-denervation and the evaluation of the response to intervention form an essential component of the surgical assessment pathway. ${ }^{8}$ Following chemodenervation assessment of weak antagonist muscle function and strengthening with targeted physiotherapy interventions can delay contracture formation and guide the surgical planning. . $^{19,20}$ 


\section{Conclusion}

The result of the survey reflects the opinion of the delegates following a session reviewing treatment options and timing of surgical intervention and as such may not reflect the wider opinion of all involved in the management of the post-stroke upper limb. However closer multi-disciplinary co-operation can ensure a standardised approach to the post-stroke upper limb and provide an opportunity for earlier surgical intervention when conservative strategies are failing to prevent joint contractures.

\section{Acknowledgments}

None.

\section{Conflicts of interest}

The authors declare there are no conflicts of interest related to the article

\section{References}

1. Namdari S, Alosh H, Baldwin K, et al. Shoulder tenotomies to improve passive motion and relieve pain in patients with spastic hemiplegia after upper motor neuron injury. J Shoulder Elbow Surg. 2011;20(5):802-806.

2. Garland DE, Thompson R, Waters RL. Musculocutaneous neurectomy for spastic elbow flexion in non-functional upper extremities in adults. $J$ Bone Joint Surg Am. 1980;62(1):108-112.

3. Buffenoir K, Rigoard P, Ferrand-Sorbets S, et al. [Retrospective study of the long-term results of selective peripheral neurotomy for the treatment of spastic upper limb]. Neurochirurgie. 2009;55(Suppl 1):S150-S160.

4. Keenan MA. Management of the spastic upper extremity in the neurologically impaired adult. Clin Orthop Relat Res. 1988;(233):116125.

5. Heijnen IC, Franken RJ, Bevaart BJ, et al. Long-term outcome of superficialis-to-profundus tendon transfer in patients with clenched fist due to spastic hemiplegia. Disabil Rehabil. 2008;30(9):675-678.

6. Keenan MA, Korchek JI, Botte MJ, et al. Results of transfer of the flexor digitorum superficialis tendons to the flexor digitorum profundus tendons in adults with acquired spasticity of the hand. J Bone Joint Surg Am. 1987;69(8):1127-1132.

7. Pappas N, Baldwin K, Keenan MA. Efficacy of median nerve recurrent branch neurectomy as an adjunct to ulnar motor nerve neurectomy and wrist arthrodesis at the time of superficialis to profundus transfer in prevention of intrinsic spastic thumb-in-palm deformity. J Hand Surg Am. 2010;35(8):1310-1316.
8. Brainin M, Norrving B, Sunnerhagen KS, et al. Poststroke chronic disease management: towards improved identification and interventions for poststroke spasticity-related complications. Int J Stroke. 2011;6(1):4246.

9. Namdari S, Keenan MA. Outcomes of the biceps suspension procedure for painful inferior glenohumeral subluxation in hemiplegic patients. $J$ Bone Joint Surg Am. 2010;92(15):2589-2597.

10. Neuhaus V, Kadzielski JJ, Mudgal CS. The role of arthrodesis of the wrist in spastic disorders. J Hand Surg Eur Vol. 2015;40(5):512-517.

11. Louis DS, Hankin FM, Bowers WH. Capitate-radius arthrodesis: an alternative method of radiocarpal arthrodesis. J Hand Surg Am. 1984;9(3):365-369.

12. Das AK, Talwalkar SC, Murali SR. Metacarpal head resection for treatment of the fingers-in-palm deformity in longstanding neurological injury. J Hand Surg Eur Vol. 2015;40(3):319-320.

13. Namdari S, Alosh H, Baldwin K, et al. Outcomes of tendon fractional lengthenings to improve shoulder function in patients with spastic hemiparesis. J Shoulder Elbow Surg. 2012;21(5):691-198.

14. Landi A, Cavazza S, Caserta G, et al. The upper limb in cerebral palsy: surgical management of shoulder and elbow deformities. Hand Clin. 2003;19(4):631-648.

15. Shin DK, Jung YJ, Hong JC, et al. Selective musculocutaneous neurotomy for spastic elbow. J Korean Neurosurg Soc. 2010;48(3):236-239.

16. Parry RH, Lincoln NB, Vass CD. Effect of severity of arm impairment on response to additional physiotherapy early after stroke. Clin Rehabil. 1999;13(3):187-198.

17. Opheim A, Danielsson A, Alt Murphy M, et al. Early prediction of long-term upper limb spasticity after stroke: part of the SALGOT study. Neurology. 2015;85(10):873-880.

18. Lindsay C, Simpson J, Ispoglou S, et al. The early use of botulinum toxin in post-stroke spasticity: study protocol for a randomised controlled trial. Trials. 2014;15:12

19. Wolfe S, Pederson W, Scott H. Kozin Green's operative hand surgery. $6^{\text {th }}$ edn. UK: Churchill Livingstone; 2011. P. 1173-1207.

20. Pandyan AD, Cameron M, Powell J, et al. Contractures in the post-stroke wrist: a pilot study of its time course of development and its association with upper limb recovery. Clin Rehabil. 2003;17(1):88-95. 